18:385-392

2. Dawkins MJR, Hull D 1964 Brown adipose tissue and the response of newborn rabbits to cold. J Physiol 172:216-238

3. Elphick MC, Johnson F, Taylor M, Davis WL 1981 An automatic oxygen consumption apparatus for small laboratory animals. Med Biol Eng Comput 19:110-116

4. Hardman MJ, Hull D, Oyesiku J 1970 The influence of birth weight and nutrition on postnatal growth of rabbits. Biol Neonate 66:306-312

5. Hardman MJ, Hull D, Oyesiku J 1970 A comparison of the growth of white and brown adipose tissue in rabbits reared under controlled conditions. Biol Neonate 16:354-361

6. Hendrickse WA, Spencer SA, Roberton DM, Hull D 1984 The calorie intake and weight gain of low birth weight infants fed on fresh breast milk or a special formulae diet. Eur $\mathrm{J}$ Paediatr, in press

7. Hervey GR, Tobin G 1983 Luxuskonsumption, diet-induced thermogenesis and brown fat: a critical review. Clin Sci 64:7-18

8. Rothwell NJ, Stock MJ 1979 A role for brown adipose tissue in diet induced thermogenesis. Nature 281:31-35

9. Rothwell NJ, Stock MJ 1982 Effects of feeding a palatable 'cafeteria' diet on energy balance in young and adult lean $(+/$ ?) Zucker rats. Br J Nutr 47:46

10. Rothwell NJ, Stock MJ 1983 Luxuskonsumption, diet-induced thermogenesis and brown fat: the case in favour. Clin Sci 64:19-23

1. Spencer SA, Hendrickse W, Roberton D, Hull D 1982 Energy intake and weight gain of very low birthweight babies fed raw expressed breast milk. $\mathrm{Br}$ Med J 285;924-926

\title{
Gestational Changes in the Germinal Matrix of the Normal Rhesus Monkey Fetus
}

\author{
NICHOLAS J. LENN and LEANNA WHITMORE \\ Departments of Neurology and Pediatrics, and the Clinical Neuroscience Research Center, University of Virginia, \\ Charlottesville, Virginia 22908
}

\begin{abstract}
To explain the reported predisposition to germinal matrix hemorrhage in premature infants, pathogenetically important morphological features of the germinal matrix should be present in the 3rd trimester and rapidly change near term. Such features were sought in this study of the germinal matrix and its vasculature in normal rhesus monkey fetuses. The matrix cells, glia, ependyma, and capillaries showed no important structural changes during the 3 rd trimester. The terminal vein tributaries were greatly enlarged by 148 days, but cellular and collagen support in their walls was minimal at this time. The latter features developed by the final days of gestation. These findings do not support a structural immaturity or specialization of the germinal matrix predisposing to germinal matrix hemorrhage. Our results, therefore, support the recent emphasis on physiological parameters in the pathogenesis and prevention of germinal matrix hemorrhage. (Pediatr Res 19:130-135, 1985)
\end{abstract}

\section{Abbreviation}

GMH, germinal matrix hemorrhage

GMH is well-known to have an age-related incidence. Premature infants have shown rates up to $40 \%(10)$, while in term babies such hemorrhages are uncommon (15). The gross correlation with the presence and subsequent disappearance of the germinal matrix near term in the human is an obvious explanation for these observations. However, it does not give any positive understanding of possible predisposing factors underlying the

Received December 15, 1983: accepted July 11, 1984.

Address for correspondence Dr. Nicholas J. Lenn, Department of Neurology, Box 394, University of Virginia, Charlottesville, VA 22908.

Supported in part by NIH Grants HD NS 08658 and RR000169. occurrence of GMH, nor any rational guidance to efforts at prevention $(4,10,12,15)$. In studies on this question, in addition to considerable attention to physiological parameters, anatomical predisposing factors have been described or suggested. Using postmortem perfusion and light microscopy, rupture along capillaries, and at capillary-venous junctions, but not at the larger veins was observed in human pathological material $(4,12)$. Several authors have suggested that anatomical features of capillary immaturity, mainly size and thinness of endothelium, may be important in the pathogenesis of $\operatorname{GMH}(4,10)$. In contrast, others have found and illustrated terminal vein rupture in similar material (6). In addition to the limited resolution of light microscopy, the preservation of the material in all such studies was necessarily compromised by the hemorrhages and by postmortem changes. Another approach is to use normal, well-fixed material from an appropriate experimental animal, rhesus monkey. Although actual hemorrhage is not present, the hypothesis can be stated as follows: if the age-related incidence of GMH is due to structural features of the germinal matrix or its vasculature, these would be present in the latter part of gestation, and disappear by term.

\section{MATERIALS AND METHODS}

The specimens were products of timed, uncomplicated pregnancies, delivered by hysterotomy under general anesthesia by the veterinary staff of the California Regional Primate Center. The 15 fetuses studied were of gestational ages (days): 60, 62, 77, $90,91,106,120$ (three cases), 132 (two cases), 133, 148 (two cases), and 162. This spans the 2nd and 3rd trimesters up to 3 days prior to term. For clarity, age differences of 1-2 days are ignored in this report. Available body and brain weights were normal, indicating that the fetuses were healthy (5). While the animals were still anesthetized, they were perfused through the heart with a mixture of $1 \%$ paraformaldehyde and $1.25 \%$ glutaraldehyde in $0.1 \mathrm{M}$ sodium cacodylate $(\mathrm{pH} \mathrm{7.2)}$ at room temper- 
ature (7). The solution was delivered through a $14-18 \mathrm{G}$ needle. External pressure of 120-150 torr was applied to the reservoir to produce a flow rate of $40-100 \mathrm{ml} / \mathrm{min}$ for the first $2 \mathrm{~min}$, and 10 $\mathrm{ml} / \mathrm{min}$ for an additional $5-10 \mathrm{~min}$. Brains were stored in the same solution in two cases, and in a solution containing twice the concentration of each aldehyde in the remainder. Samples of the germinal matrix were dissected as coronal blocks guided by the location of the foramen of Monro and adjoining structures. The tissue was rinsed in buffer, and then exposed to $2 \%$ osmium tetroxide in $0.2 \mathrm{M}$ sodium cacodylate with $0.2 \mathrm{M}$ sucrose and $1.5 \%$ potassium ferricyanide for $2 \mathrm{~h}$. Block staining with uranyl acetate was followed by dehydration and embedding in Durcupan. Thicker sections were stained with toluidine blue for light microscopy. Ultrathin sections were contrasted with lead citrate and uranyl acetate prior to examination in a Hitachi EM 12. Photomicrographs were obtained at original magnifications of $1,000-30,000$

Stereological studies were performed on photomicrographs of unselected capillaries obtained from specimens at 62, 120, 148, and 162 days gestation. They were measured by the area to area method using a Zeiss Videoplan computer based image analysis system. Nonparametric tests of significance (U test) were performed on the same system.

\section{RESULTS}

Germinal matrix. At 60 days the germinal matrix of the forebrain, the ganglionic eminence, is a prominent mass protruding into the lateral ventricle. This portion of the germinal matrix gives rise to a number of telencephalic structures, and to the pulvinar (14). It consists of a homogeneous population of small germinal cells, with mitoses in the ventricular zone (Fig. 1). The ganglionic eminence contains numerous small vessels which are variable in size, from $5-100 \mu$ (Fig. 1). These vessels are as close as $0.1 \mathrm{~mm}$ to the ventricular surface. At subsequent ages the number of germinal cells in this region decreases. By 120 days they are no longer confluent, but rather are intermixed with subependymal glial cells or grouped in perivascular areas.
Mitoses are no longer seen. By 162 days, only perivascular germinal cells remain.

Fibrillary astrocytes appear at 90 days. By 120 days they form an extensive subependymal population easily distinguished from the germinal cells. Vascular end-feet with gap junctions are present by 120 days. The apparent loose character of the subependymal zone in late gestation has been commented on by various authors (3), suggesting low tissue support for the enlarging vessels. However, the known swelling of glia to even slight, much less major, delays in fixation leads to caution. Our specimens at 120 days, for instance, show that in the best fixed material this sponginess is not present. The artifactual character of the sponginess was confirmed by standard electron microscopic criteria. Parenchymal glia show parallel changes at these ages.

Ependyma. An ependymal layer is present on the ventricular surface of the germinal matrix at all ages studied. At 60 days, the ependymal cells are flattened (Fig. 2). They have occasional microvilli on their luminal surface, but no cilia. Intercellular zonula adherens are present, associated with tonofilaments (Fig. 2 , inset). By 120 days, the ependyma has become a mature high cuboidal epithelium. The luminal surface of the ependymal cells is densely covered with microvilli, and numerous mature cilia are present. Zonula adherens are again noted between ependymal cells (Fig. 3, inset). There is no further change in the ependyma at later ages.

Capillaries and their endothelium. Vessels whose wall consisted of a single endothelial layer have been identified as capillaries, even if one or two pericytes incompletely surrounded the endothelium. Vessels with muscularis and those with complete or multicellular adventitia and collagen were excluded from this category in accordance with standard nomenclature (1). Capillaries varied in diameter between 5 and $100 \mu$. At 60 days, the capillary endothelium was 5-7 $\mu \mathrm{m}$ thick (Fig. 4). The endothelial cells formed tight junctions with typical projections at points of contact. No vessels without lumina were present. Qualitatively, the only change in individual capillaries at subsequent ages was an increase in endothelial thickness (Fig. 5). In contrast to the

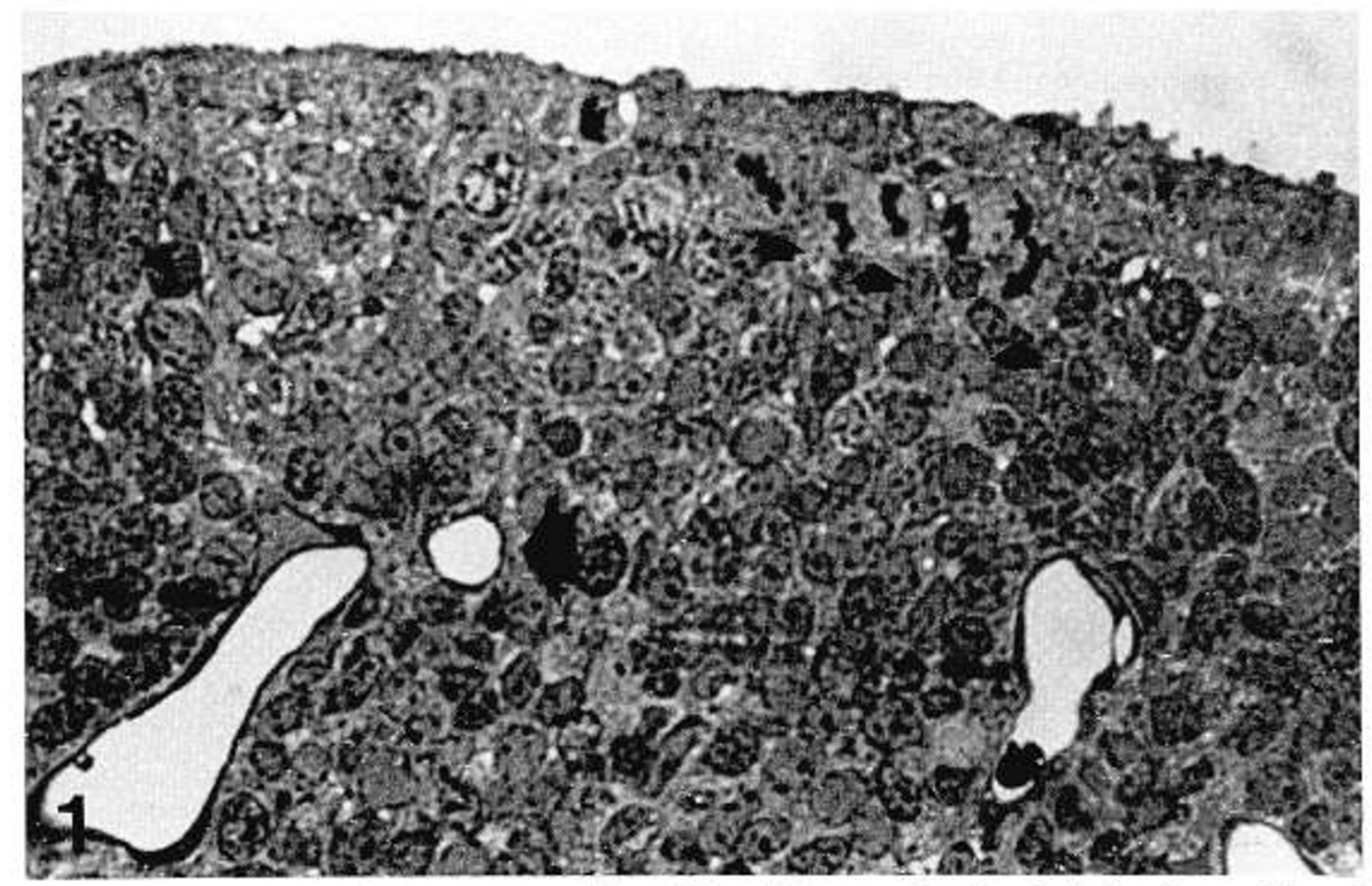

Fig. 1. Light micrograph showing part of the germinal matrix in a 60-day-old rhesus monkey fetus, including the ventricular surface (top). The germinal cells present a homogeneous population, except for several cells undergoing mitosis near the ventricular surface (arrows). The capillaries (large arrow) are variable in size, but are otherwise similar in structure. Plastic embedded, $1 \mu \mathrm{m}$ section, toluidine blue stain. $\times 108$. 


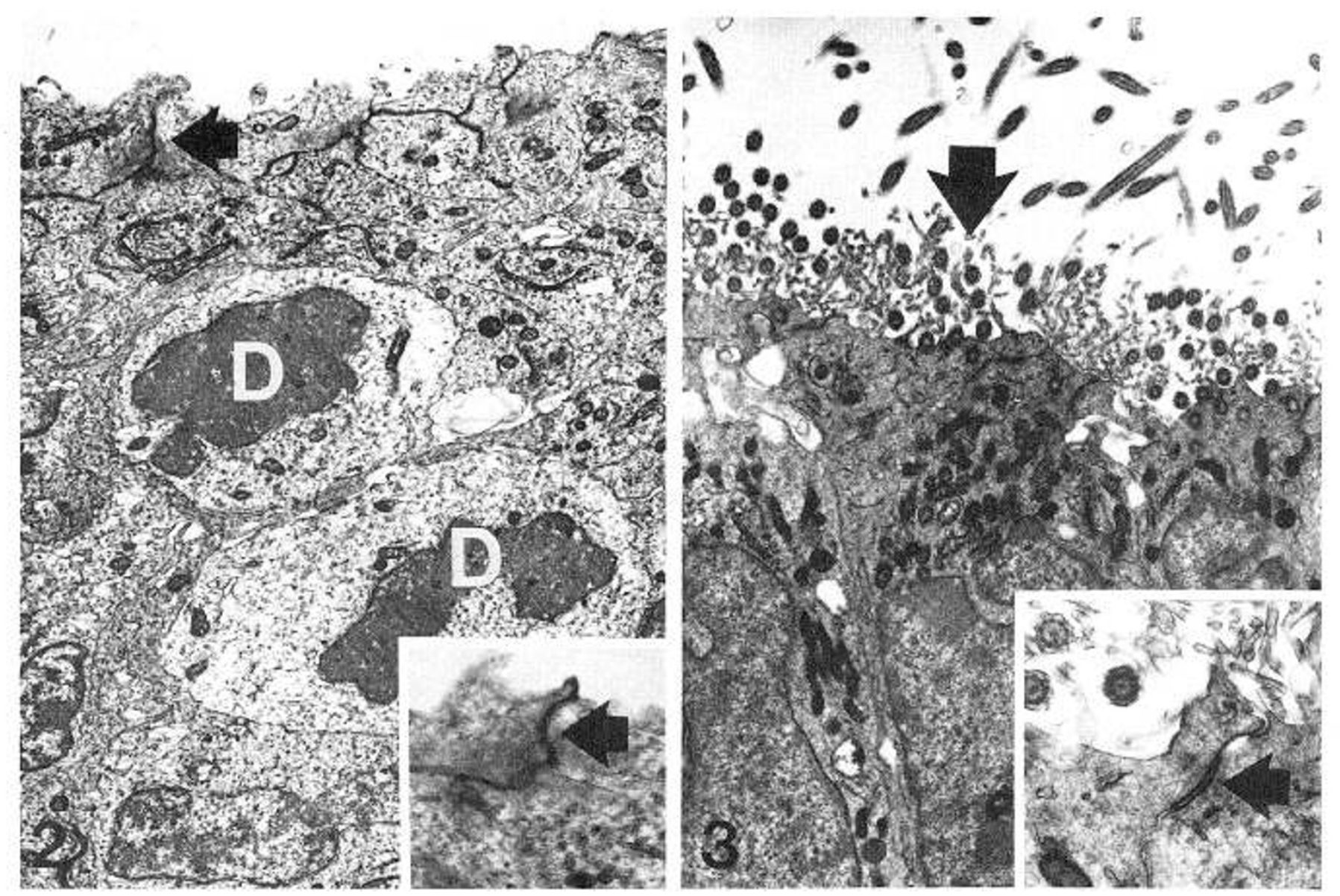

Fig. 2. Electron micrograph showing part of the germinal matrix of a 60 -day fetus, including the ventricular surface (top). The ependymal layer consists of flattened cells with zonula adherens and tonofilaments (top, left; and inset; arrows). The two daughter cells $(D)$ resulting from a recently completed mitosis are seen amongst portions of several germinal cells. $\times 9,000$; insert $\times 25,000$.

Fig. 3. Electron micrograph showing ependyma of a 120-day fetus. The ependyma is now a high cuboidal epithelium. The apical portions and ventricular surface (top) of several cells are shown. There are numberous cilia and microvilli (large arrow). Zonula adherens are present near the luminal surface (inset, arrow). $\times 9,000$ inset $\times 25,000$.

adjacent areas of corpus striatum and thalamus where the number and density of capillaries both increased markedly in late gestation, these decreased in the germinal matrix area.

Stereology on an unselected sample of capillaries from age 62 162 days showed no change in the ratio of mitochondrial volume to endothelium which is a measure of metabolic activity. The values ranged from $2.66 \pm 0.4 \%$ to $4.2 \pm 0.4 \%$. These values are comparable to systemic, but considerably less than cerebral vessels in adult rats (11). The ratio of lumen volume to endothelial volume was also measured. It did not increase between 62 and 120 days $(p>0.1)$, but did increase between 120 and 148 days, from $1.7 \pm 0.22$ to $5.2 \pm 0.4(p<0.01)$. A smaller decrease between 148 and 162 days from $5.2 \pm 0.4$ to $4.2 \pm 0.4$ has a lower probability of significance $(p<0.05)$. The 162-day value is also greater than that at 120 days $(p<0.01)$. This pattern is in keeping with previous observations $(2,9)$. The thickness of basement membrane around these vessels averaged $20 \mathrm{~nm}$ at 62 days, and increased to $40 \mathrm{~nm}$ by 120 days, with no significant change subsequently $(p>0.1)$. Therefore, this change is also completed prior to the time of concern for GMH.

Terminal vein and its major tributaries. These vessels first appear as several larger but still thin-walled subependymal venules at 132 days gestation. In several specimens at 148 days, despite luminal diameters up to $400 \mu$, the walls of these veins consist only of the endothelium, scant pericytes, and very scant collagen (Fig. 6A). At 162 days, in contrast, the vein of similar size and location has multiple layers of pericytes and dense collections of collagen (Fig. $6 B$ ). Thus, enlargement of the terminal vein and its tributaries during the time of interest for
GMH precedes any substantial thickening of the wall, and the production of a thick collagen-strengthened wall around these veins coincides with the decrease in risk of GMH near term.

\section{DISCUSSION}

The relevance of the present observations to GMH in human neonates rests on several issues. The use of well-fixed normal material to test the hypothesis of the present study clearly is necessary. The use of rhesus monkeys and the validity of extrapolation from this species to humans is supported by the major similarities of neuroembryology in these two species $(13,14)$. However, despite a few attempts, GMH does not appear to have been produced in rhesus monkeys. The most direct support for the proposed extrapolation is the similarity between the earlier specimens in the present study and the early human material studied electron microscopically by Povlishock et al. (13).

There is some difficulty in relating the time of predilection for GMH in humans, 24-36 wk postconception, to specific gestational ages in rhesus monkeys. Although there are detailed and well-correlated staging systems for the embryonic period proper in these two species, there is no staging system for the fetal period of either. Also there are no detailed comparisons available for this period which encompasses the latter two-thirds of gestation. However, there is a comparable development of the nervous system at the beginning of the fetal period, which occurs at $8 \mathrm{wk}$ in humans and 45 days in rhesus monkeys (5), and at birth. That is, there is a general equivalence of the 32 -wk fetal period in humans and the 17-wk fetal period in the rhesus. This implies 

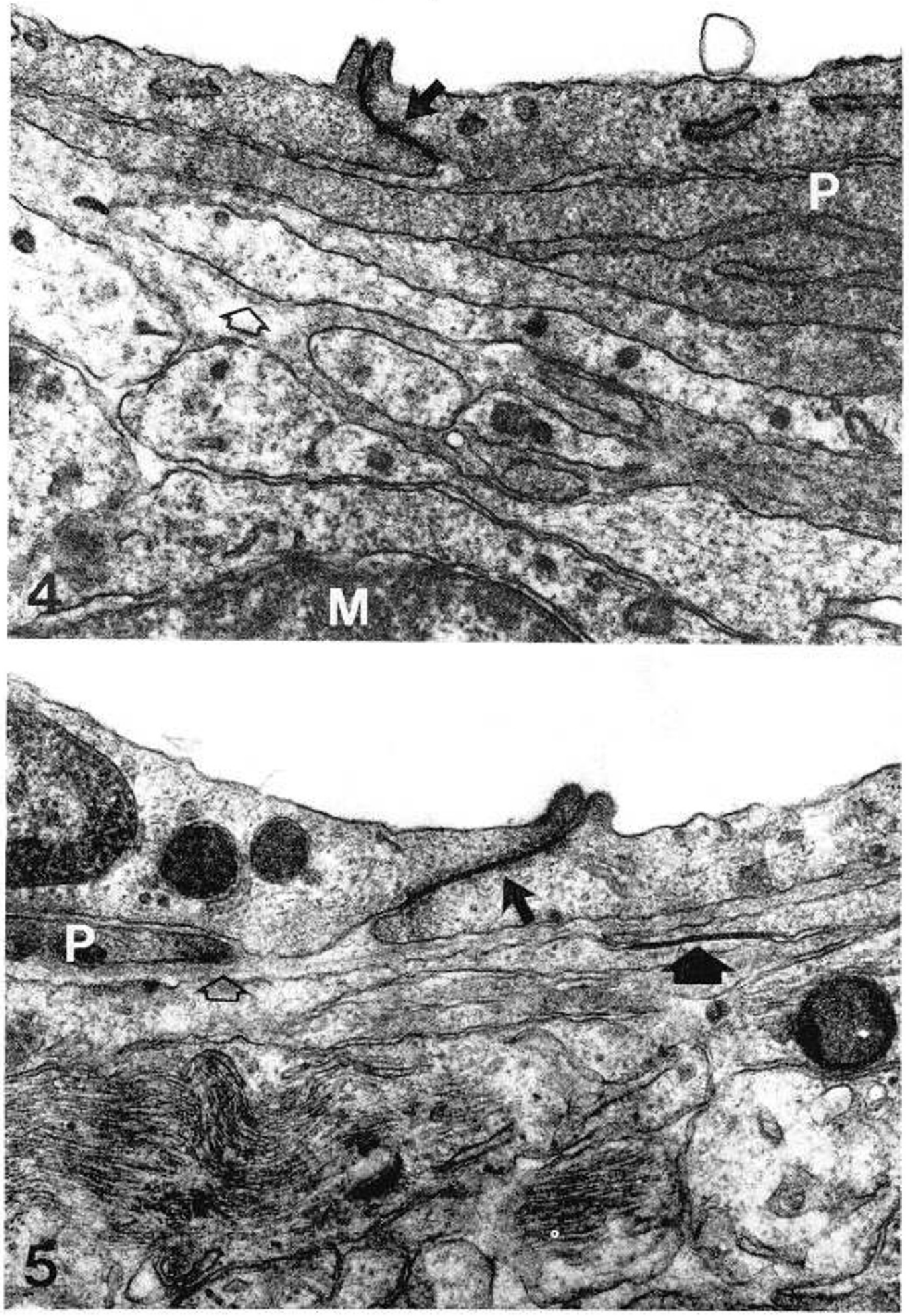

Fig. 4. Electron micrograph showing capillary endothelium at 60 days. The lumen is at top. An intercellular tight junction with typical projections is seen (arrow). Pericyte processes $(P)$, basement membrane (open arrow) and portions of matrix cells $(M)$ are below. $\times 48,000$.

Fig. 5. Electron micrograph showing capillary endothelium at 162 days. The lumen is at top. An intercellular tight junction with typical projections is seen (arrow). Pericyte process (left, P), basement membrane (open arrow) and glial processes are seen. Glial filaments are present in processes at lower left and center. A gap junction between two glial processes is present at large arrow. There are no qualitative differences in the endothelium compared to the 60 -day fetus (Fig. 4). $\times 48,000$.

an exponential rather than a linear relationship, so that development in rhesus fetuses would be somewhat ahead of that in human fetuses through most of this time. Another relevant criterion for the present purposes is the presence of the ganglionic eminence. This structure is absent in human fetuses by about 36 wk and in rhesus fetuses after 148 days. These considerations indicate that the interval from $24-36 \mathrm{wk}$ gestation in humans corresponds to 110-150 days of rhesus gestation.

The methodology used in the present study was designed to achieve optimal fixation for electron microscopic study. The 


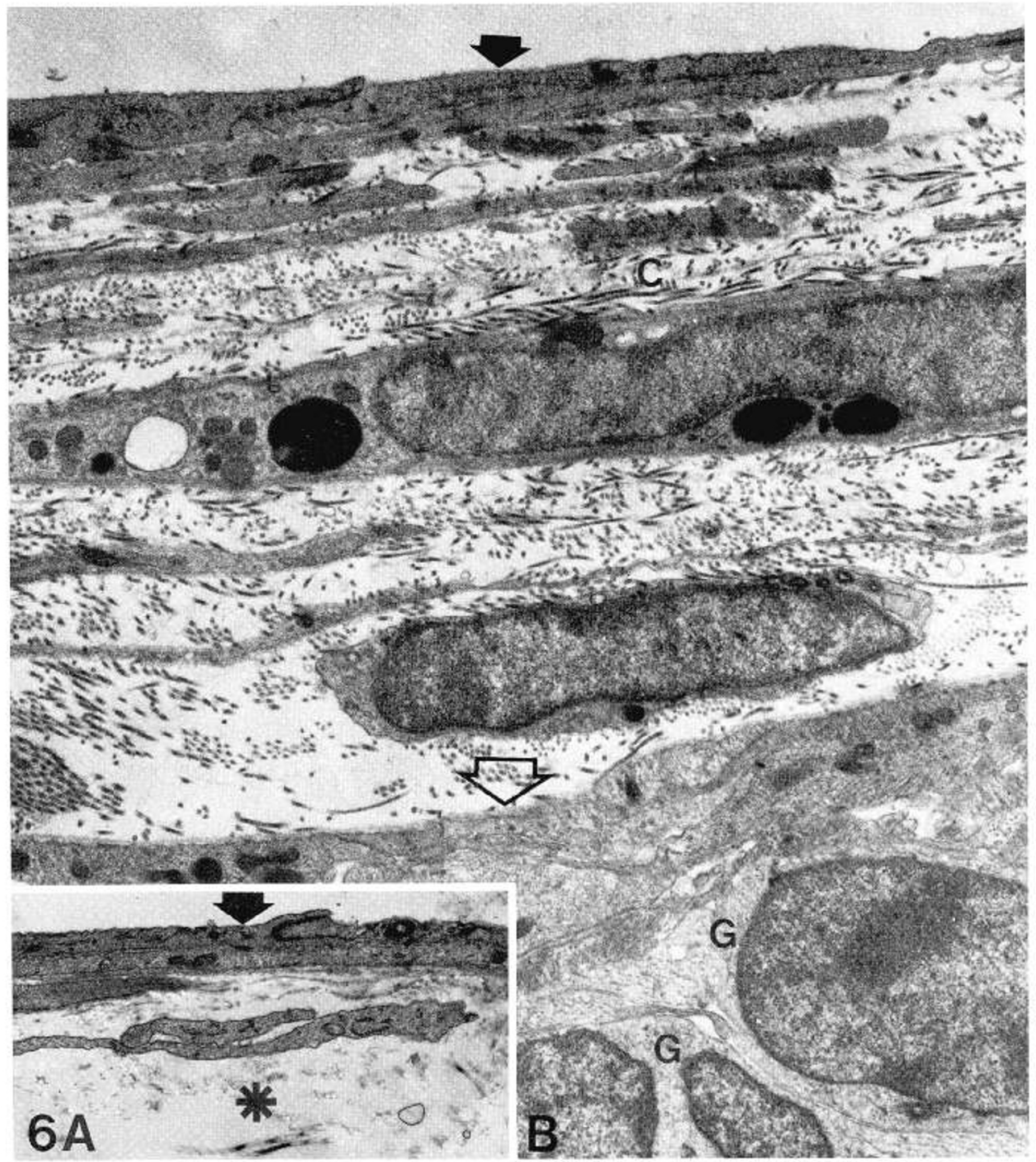

Fig. 6. Electron micrographs showing wall of a large terminal vein tributary at 148 days $(A)$ and at 162 days $(B)$. $A$ and $B$ are at the same magnification. In both the endothelium (arrow) is near the $t o p$, with the lumen above. In $A$ scanty perivascular cells and minimal collagen form the vessel wall. The perivascular space $\left(^{*}\right)$ may be enlarged artifactually, but there is no other vein wall structure. In $B$, there are multiple layers of perivascular cells intermixed with collagen $(C)$. A basement membrane separates the perivascular space from the germinal matrix (open arrow). Portions of several matrix cells $(G)$ are present at the bottom of the figure. A and $\mathrm{B}, \times 15,000$.

sampling of germinal matrix was based on surrounding landmarks, including the foramen of Monro and the thalamus. However, direct comparability between specimens is complicated by the rapid growth and development of both the brain and its vasculature, as well as the regression of the ganglionic eminence. Furthermore, sampling rather than systematic study of all levels of the vasculature was performed. This limitation has been considered in formulating our conclusions. It is less of a con- 
founding factor in comparing the vein walls between 148 and 162 days of gestation which is the most problematic comparison. Therefore, the present material is generally satisfactory for the purposes of this study. Good to excellent fixation has been obtained in the ganglionic eminence and in the brain, several regions of which have been studied in various laboratories (8). Recognition and classification of cell types, and various types of blood vessels has not been a problem. No unanticipated or new anatomical features were uncovered. The stereological analysis was performed by standard methods using a computer-based digitizing system.

The use of time correlations to demonstrate pathogenesis is far short of proof. The strongest case is actually made with regard to some of the negative correlations of the present study. For example, the ependyma and capillary endothelium were both advanced in their development prior to 120 days of gestation. After this time only the lumen to endothelial ratio changes further, and this is completed prior to 148 days. This lack of correlation to the time of predilection for GMH makes it very unlikely that these structures have anatomical properties correlating with GMH.

On the other hand, some care is appropriate in interpreting the main positive correlation found in the present study. This is the observed enlargement of the terminal vein and its tributaries during the time of predilection for GMH preceding their acquiring a well-developed, collagen reinforced adventitia. The adventitia does develop rapidly and markedly around these vessels shortly before term, correlating nicely with the expected time of decreasing predilection for GMH. However, these are correlations and a pathogenetic role can only be considered possible and suggestive. It would be unwise to ignore the evidence against rupture of these veins in human cases of GMH (12), even though it is contradicted by others (6). With this possible exception, the present findings contradict previous suggestions of structural factors in the pathogenesis of GMH, such as immaturity of the capillaries $(4,10,12)$. Thus, there is only one suggestive anatomical feature of the germinal matrix which might predispose to
GMH, and no unsuspected or apparently insurmountable features which stand in the way of preventing GMH by attention to physiological parameters $(10,12,15)$.

Acknowledgments. Mrs. Viviana Wong, Dr. V. Vijayan, Dr. Andrew Hendrickx, and his staff assisted in obtaining the specimens.

\section{REFERENCES}

1. Dahl E 1973 The fine structure of intracerebral vessels. Z Zellforsch $145: 577$

2. Donahue S, Pappas GD 1961 The fine structure of capillaries in the cerebral cortex of the rat at various stages of development. Am J Anat 108:331

3. Gruenwald $P 1951$ Subependymal cerebral hemorrhage in premature infants, and its relation to various injurious influences in birth. Am J Obstet Gynecol 61:1285

4. Hambleton G, Wigglesworth JS 1976 Origin of intraventricular haemorrhage in the preterm infant. Arch Dis Child 51:651

5. Hendrickx, AG, Sawyer, RH: Embryology of rhesus monkey. In: Hendrickx AG (ed) The Rhesus Monkey, Vol II, Management, Reproduction and Pathology. Academic Press, New York, pp 141-169

6. Larroche, J-C 1977 Developmental Pathology of the Neonate. Excerpta Medica, Amsterdam, pp 319-398

7. Lenn, NJ, Beebe, B 1976 Controlled pressure perfusion fixation. Microsc Acta 17:234

8. Lenn, NJ, Wong, V 1982 Crest synapse structure and location within the interpeduncular nucleus of the near-term rhesus monkey fetus. Ann Neurol $12: 222$

9. Majno, G 1965 Ultrastructure of the vascular membrane. In: Hamilton WP Dow P (eds) Handbook of Physiology Sect 2, Vol III. American Physiology Society, Washington, DC, pp 2293-2375

10. Milligan, DWA 1980 Failure of autoregulation and intraventricular haemorrhage in preterm infants. Lancet 1:896

11. Oldendorf, WH, Cornford, ME, Brown, WJ 1978 The large apparent work capability of the blood-brain barrier: a study of the mitochondrial content of capillary endothelial cells in brain and other tissues of the rat. Ann Neurol $1: 409$

12. Pape, KE, Wigglesworth, JS 1979 Haemorrhage, Ischaemia and the Perinatal Brain. Heinemann Medical Books, London, pp 118-132

13. Povlishock, JT, Martinez, AJ, Moossy, J 1977 The fine structure of blood vessels of the telencephalic germinal matrix in the human fetus. Am $J$ Ana 149:439

14. Rakic, P 1977 Development of the pulvinar in primates. In: Cooper JS, Rakic P (eds) The Pulvinar. Charles C Thomas, Springfield, IL, pp 1-21

15. Volpe, JJ 1981 Neonatal intraventricular hemorrhage. N Engl J Med 304:886 